University of Wollongong

Research Online

Australian Institute for Innovative Materials -

Papers

Australian Institute for Innovative Materials

$1-1-2018$

Controlled synthesis of mesoporous nitrogen-doped carbons with highly ordered two-dimensional hexagonal mesostructures and their chemical activation

\author{
Abeer Enaiet Allah \\ National Institute For Materials Science, Beni-Suef University \\ Haibo Tan \\ National Institute For Materials Science, Waseda University \\ Xingtao Xu \\ Hohai University \\ Ahmed Farghali \\ Beni-Suef University \\ Mohamed Khedr \\ Beni-Suef University
}

See next page for additional authors

Follow this and additional works at: https://ro.uow.edu.au/aiimpapers

Part of the Engineering Commons, and the Physical Sciences and Mathematics Commons

Research Online is the open access institutional repository for the University of Wollongong. For further information contact the UOW Library: research-pubs@uow.edu.au 


\title{
Controlled synthesis of mesoporous nitrogen-doped carbons with highly ordered two-dimensional hexagonal mesostructures and their chemical activation
}

\author{
Abstract \\ Ordered mesoporous nitrogen-doped carbon (OMNC) materials are considered as the most promising \\ material for supercapacitors. In this study, a highly ordered two-dimensional (2D) hexagonal \\ mesostructured polymer was synthesized through a facile assembly of triblock polymer micelles and \\ phenol-melamine/formaldehyde resin via an organic-organic assembly process in aqueous solution. After \\ calcination, the novel OMNC materials with 2D hexagonal mesostructures were obtained. By further $\mathrm{KOH}$ \\ activation, the surface area and the porosity of the OMNC significantly improved, and the internal \\ mesoporous structures were maintained. The activated OMNC-800A displayed a specific capacitance as \\ high as $475.75 \mathrm{~F} \mathrm{~g}-1$ at $0.5 \mathrm{~A} \mathrm{~g}-1$ with an outstanding cycling stability (over $100 \%$ capacitance retention \\ during 2000 cycling tests at $100 \mathrm{mV} \mathrm{s}-1$ ). These results confirm that the tubular mesochannels inside the \\ $\mathrm{OMNC}$ are very beneficial in providing an accessible path for diffusion of the electrolyte, thereby \\ improving the specific capacitance of OMNC at a high current density.

\section{Disciplines} \\ Engineering | Physical Sciences and Mathematics

\section{Publication Details} \\ Allah, A. Enaiet., Tan, H., Xu, X., Farghali, A. A., Khedr, M. Hamdy., Alshehri, A. Ali., Bando, Y., Kumar, N. \\ Ashok. \& Yamauchi, Y. (2018). Controlled synthesis of mesoporous nitrogen-doped carbons with highly \\ ordered two-dimensional hexagonal mesostructures and their chemical activation. Nanoscale, 10 (26), \\ 12398-12406.
}

\section{Authors}

Abeer Enaiet Allah, Haibo Tan, Xingtao Xu, Ahmed Farghali, Mohamed Khedr, Abdulmohsen Alshehri, Yoshio Bando, Nanjundan Kumar, and Yusuke Yamauchi 


\title{
Nanoscale
}

\section{Check for updates}

Cite this: Nanoscale, 2018, 10, 12398

\section{Controlled synthesis of mesoporous nitrogen-doped carbons with highly ordered two-dimensional hexagonal mesostructures and their chemical activation $\uparrow$}

\author{
Abeer Enaiet Allah, $\psi^{\mathrm{a}, \mathrm{b}}$ Haibo Tan, (D) $* *^{\mathrm{a}, \mathrm{c}}$ Xingtao $\mathrm{Xu},{ }^{* \mathrm{~d}}$ Ahmed A. Farghali, \\ Mohamed Hamdy Khedr, ${ }^{\mathrm{e}}$ Abdulmohsen Ali Alshehri, ${ }^{f}$ Yoshio Bando, ${ }^{a, g}$ \\ Nanjundan Ashok Kumar (D) ${ }^{\mathrm{h}}$ and Yusuke Yamauchi (D)*h,i
}

\begin{abstract}
Ordered mesoporous nitrogen-doped carbon (OMNC) materials are considered as the most promising material for supercapacitors. In this study, a highly ordered two-dimensional (2D) hexagonal mesostructured polymer was synthesized through a facile assembly of triblock polymer micelles and phenol-melamine/formaldehyde resin via an organic-organic assembly process in aqueous solution. After calcination, the novel OMNC materials with 2D hexagonal mesostructures were obtained. By further $\mathrm{KOH}$ activation, the surface area and the porosity of the OMNC significantly improved, and the internal mesoporous structures were maintained. The activated OMNC-800A displayed a specific capacitance as high as $475.75 \mathrm{~F}$ $\mathrm{g}^{-1}$ at $0.5 \mathrm{~A} \mathrm{~g}^{-1}$ with an outstanding cycling stability (over 100\% capacitance retention during 2000 cycling tests at $100 \mathrm{mV} \mathrm{s}^{-1}$ ). These results confirm that the tubular mesochannels inside the OMNC are very beneficial in providing an accessible path for diffusion of the electrolyte, thereby improving the specific capacitance of OMNC at a high current density.
\end{abstract}

Received 2nd April 2018, Accepted 23rd May 2018

DOI: $10.1039 / c 8 n r 02647$ e

rsc.li/nanoscale controllable mesoporous structures, etc. ${ }^{1,2}$ These materials are ideal candidates for a myriad of applications, particularly electrochemical energy conversion and storage. $^{3-5}$ To date, hard- and soft-templating approaches have been employed for the preparation of ordered mesoporous (OMC) carbon materials with controlled morphology, uniform pore structures and narrow pore size distribution. Hard-templating approaches including mesoporous silica have been utilized for the synthesis of mesoporous carbon materials. ${ }^{6-8}$ However, the hard-templating approach is a multistep process (i.e., preparation of ordered templates, replication of mesopores with appropriate carbon precursors, carbonization, and removal of the template matrix). In this case, the rational design and control of templates are the key factors for producing highquality mesoporous materials which are slightly complex, time and cost consuming, and unfavorable for mass production. In contrast, the soft-templating method is a straightforward approach which is dependent on the self-assembly of the organic compounds and block copolymers into ordered mesostructured composites in a oriented manner through a chemical interaction. ${ }^{9-13}$ Interestingly, the morphology, porosity, and structure could be tuned by controlling the synthetic conditions, such as the concentration of the surfactant, $\mathrm{pH}$, and carbonization temperature. The morphological control will endow the product with an excellent chemical activity, 
mechanical stability, good electrical conductivity, and high surface area, which have been considered as the essential requirements for electrode materials used in energy storage applications. ${ }^{14,15}$

Supercapacitors have received considerable attention in customer electronics, communication, and transportation systems because of their superior power density and long cycling durability. ${ }^{16,17}$ Research efforts are still directed toward increasing the power density and extending the long-term durability of electrodes to fulfill the requirements of high power delivery/uptake in portable electronics and other equipment with high power demands. ${ }^{18,19}$ Controlling pore size distribution is another crucial factor for carbon electrodes to boost high power density by exposing the accessible surface and providing a diffusion path for electrolytes. ${ }^{20-23}$ Besides, it was also proved that not only the high surface area but also other parameters, such as nitrogen doping ${ }^{24}$ and carbon particle size, ${ }^{25}$ are crucial for improving the specific capacitance and the rate performance of carbon electrodes in supercapacitors. Therefore, it is expected that the fabrication of ordered mesoporous nitrogen-doped carbon (OMNC) may integrate the advantages of OMC with nitrogen doping. We can expect that the presence of tubular mesochannels is also beneficial to exposing the accessible surface area and accelerating the diffusion of electrolytes. However, synthesizing OMNCs with $2 \mathrm{D}$ hexagonal mesostructures still remains a challenge due to the complications in optimizing the synthetic conditions. $^{26-31}$

In this study, a highly ordered two-dimensional (2D) hexagonal mesostructured polymer was synthesized through a facile assembly of triblock polymer micelles and phenol-melamine/ formaldehyde resin via an organic-organic assembly process in aqueous solution. The symmetric triblock polymer Pluronic P123 was used as a mesopore-direct agent, and phenol/formaldehyde and melamine/formaldehyde resin were used as carbon and nitrogen sources, respectively. After calcination, the as-prepared OMNC possessed uniformly sized mesopores and plentiful tubular mesochannels. With further $\mathrm{KOH}$ activation at high temperatures, the morphology and ordered mesopores of OMNC were still maintained, and they also showed an ultrahigh surface area (over $2400 \mathrm{~m}^{2} \mathrm{~g}^{-1}$ ) mainly by creating abundant micropores. As a supercapacitor electrode, the activated OMNC showed outstanding specific capacitance as high as $475.75 \mathrm{~F} \mathrm{~g}^{-1}$ at $0.5 \mathrm{~A} \mathrm{~g}^{-1}$ and remarkable long-term stability.

\section{Experimental section}

\subsection{Chemicals}

Pluronic P123 (PEG-PPG-PEG), melamine, and 5.0 wt\% Nafion perfluorinated resin solution were purchased from SigmaAldrich. Phenol, formaldehyde (37 wt\%), $0.1 \mathrm{M}$ sodium hydroxide, and $1 \mathrm{M} \mathrm{H}_{2} \mathrm{SO}_{4}$ were purchased from Nacalai Tesque, Inc. All the chemicals were of analytical grade and used as received.

\subsection{Preparation of ordered mesoporous nitrogen-doped carbon (OMNC)}

Firstly, the mesostructured polymer was prepared via an organic-organic assembly process in aqueous solution with the assistance of the triblock copolymer Pluronic P123. Phenol-melamine/formaldehyde resin (P-M/F) was added as carbon and nitrogen sources. Typically, $0.28 \mathrm{~g}$ of phenol and $0.19 \mathrm{~g}$ of melamine were dissolved in $1.5 \mathrm{~mL}$ of formaldehyde aqueous solution (37 wt\%) under stirring at $60{ }^{\circ} \mathrm{C}$, leading to a colorless transparent solution after $5 \mathrm{~min}$. Then, $2.0 \mathrm{~mL} 0.1 \mathrm{M}$ $\mathrm{NaOH}$ solution was added into the above clear solution and stirred at $70{ }^{\circ} \mathrm{C}$ for $30 \mathrm{~min}$. After this, $10.0 \mathrm{~mL}$ P123 aqueous solution (containing $0.325 \mathrm{~g}$ of P123) was added and the solution was stirred at $65{ }^{\circ} \mathrm{C}$ for $2 \mathrm{~h}$. Subsequently, $25.0 \mathrm{~mL}$ of distilled water was added to the mixture and the diluted solution was further kept at $65{ }^{\circ} \mathrm{C}$ for another $10 \mathrm{~h}$ under mild stirring, resulting in a dark-red solution. After that, $10.0 \mathrm{~mL}$ of the obtained dark-red solution was transferred in a Teflon liner under vigorous stirring. The $\mathrm{pH}$ value was adjusted to $c a$. 9.3 by adding an appropriate volume of $0.1 \mathrm{M} \mathrm{NaOH}$ solution. The Teflon liners were transferred into autoclaves $(100 \mathrm{~mL})$ for a hydrothermal reaction at $130{ }^{\circ} \mathrm{C}$ for $20 \mathrm{~h}$ with a ramping rate of $1{ }^{\circ} \mathrm{C} \mathrm{min}^{-1}$. The obtained precipitate was collected by centrifugation (14000 rpm for $5 \mathrm{~min}$ ) and washed with water and ethanol three times. After drying at $60{ }^{\circ} \mathrm{C}$, the mesostructured polymers were obtained. The synthetic conditions (e.g., concentration of $\mathrm{P} 123$ and $\mathrm{pH}$ ) for controlling the morphology of the precursor were carefully investigated and the corresponding products were denoted as N/C- $x(x=1,2,3,4$, and 5). The detailed synthesis parameters are listed in Table 1 . The as-made precursor was carbonized at controlled temperatures for $4 \mathrm{~h}$ under a $\mathrm{N}_{2}$ atmosphere. The ramping rate of the calcination process was $5{ }^{\circ} \mathrm{C} \min ^{-1}$. The pre-carbonized ordered mesoporous nitrogendoped carbons were denoted as OMNC- $T$, where $T$ represents the calcination temperatures $\left(700,800\right.$, and $\left.900{ }^{\circ} \mathrm{C}\right)$.

\section{3. КОH activation of OMNC-T}

$25.0 \mathrm{mg}$ of OMNC- $T$ was mixed with $100.0 \mathrm{mg}$ potassium hydroxide $(\mathrm{KOH})$. Then, the mixture was dispersed in $5 \mathrm{~mL}$ of deionized water under sonication for $30 \mathrm{~min}$. The slurry was dried at $100{ }^{\circ} \mathrm{C}$ overnight, following by thermal treatment at $800{ }^{\circ} \mathrm{C}$ for $2 \mathrm{~h}$ under flowing $\mathrm{N}_{2}$ with a ramping rate of $5{ }^{\circ} \mathrm{C}$ $\mathrm{min}^{-1}$. After cooling to room temperature, the resulting powder was washed with deionized water to remove $\mathrm{KOH}$ completely. Finally, the activated OMNC- $T$ was dried in an oven at $80{ }^{\circ} \mathrm{C}$ and labeled as OMNC-TA.

Table 1 Different preparation conditions with changing P123 concentrations or $\mathrm{pH}$ values

\begin{tabular}{lcc}
\hline Samples & Concentration of P123 $\left(\mathrm{mg} \mathrm{mL}^{-1}\right)$ & $\mathrm{pH}$ values \\
\hline N/C-1 & 32.5 & 8.5 \\
N/C-2 & 32.5 & 9.3 \\
N/C-3 & 32.5 & 10.0 \\
N/C-4 & 10.0 & 9.3 \\
N/C-5 & 100.0 & 9.3
\end{tabular}




\subsection{Characterization}

The morphology of the samples was characterized by using a field emission scanning electron microscope (SEM, HITACHI SU-8230) operating at $5.0 \mathrm{kV}$. A transmission electron microscope (TEM, JEOL JEM-2100F) operated at $200 \mathrm{kV}$ was employed to investigate the inner mesoporous structure. Small angle X-ray scattering (Rigaku NANO Viewer; $\mathrm{Cu} \mathrm{K} \alpha$ radiation; $40 \mathrm{kV}$, $30 \mathrm{~mA}$ ) equipped with a camera length of $700 \mathrm{~mm}$ was used to evaluate the pore-to-pore distance. Wide-angle X-ray diffraction (XRD) patterns were acquired with a Rigaku Rint 2000 X-ray diffractometer using monochromated $\mathrm{Cu} \mathrm{K \alpha}$ radiation $(40 \mathrm{kV}$, $40 \mathrm{~mA}$ ) at a scanning rate of $1^{\circ} \mathrm{min}^{-1}$. Nitrogen adsorption-desorption isotherms were obtained using a BELSORP-mini (Bel Japan, Inc.) at $77 \mathrm{~K}$. The specific surface area (SSA) was evaluated by the multipoint Brunauer-Emmett-Teller (BET) method at a relative pressure from 0.05 to 0.3 based on the adsorption data. The chemical state of nitrogen was investigated using X-ray photoelectron spectroscopy (PHI Quantera SXM) with Al $\mathrm{K} \alpha$ radiation $(20 \mathrm{kV}, 5 \mathrm{~mA})$. The binding energy shift was calibrated using the $\mathrm{C} 1 \mathrm{~s}$ level at $284.5 \mathrm{eV}$.

\subsection{Electrochemical measurements}

The as-prepared OMNC-T and OMNC-800A were ground before preparing inks. In a typical experiment, $2.0 \mathrm{mg}$ of sample was dispersed in $400 \mu \mathrm{l}$ of $1: 2(\mathrm{v} / \mathrm{v})$ isopropanol/water mixed solvent (containing $20 \mu \mathrm{L}$ of $5.0 \mathrm{wt} \%$ Nafion solution) under sonication for at least $30 \mathrm{~min}$ to form a homogeneous ink. After that, $200 \mu \mathrm{L}$ of the suspension was dropped onto flexible graphite paper (thickness: $1 \mathrm{~mm}$ ) with an area of $1 \times 1 \mathrm{~cm}^{2}$ and dried at $60{ }^{\circ} \mathrm{C}$. The mass loading was $1.0 \mathrm{mg} \mathrm{cm}^{-2}$ and the thickness of the electrode film was found to be around 15-25 $\mu \mathrm{m}$. In addition, we choose flexible graphite paper as the current collector because of its excellent electrical conductivity as well as its negligible capacitive performance (usually below $1 \mathrm{~F} \mathrm{~g}^{-1}$ ), which would contribute little to the total capacitance of the prepared working electrode. All electrochemical measurements were carried out on a CHI $660 \mathrm{E}$ instrument. In a three-electrode system, the electrochemical performances of the as-made OMNC- $T$ and OMNC-800A electrodes were investigated by cyclic voltammetry $(\mathrm{CV})$ and galvanostatic charge-discharge (GCD) in $1 \mathrm{M} \mathrm{H}_{2} \mathrm{SO}_{4}$, with a platinum wire as the counter electrode and an $\mathrm{Ag} / \mathrm{AgCl}$ electrode as the reference electrode. The specific capacitance of the asmade electrodes was calculated from the GCD curves. The test of long-term stability for OMNC-800A was conducted by cycling between 0.0 and $0.8 \mathrm{~V}$ vs. $\mathrm{Ag} / \mathrm{AgCl}$ in $1 \mathrm{M} \mathrm{H}_{2} \mathrm{SO}_{4}$ at a scan rate of $100 \mathrm{mV} \mathrm{s}^{-1}$.

In general, the gravimetric specific capacitance $\left(C_{\mathrm{g}}, \mathrm{F} \mathrm{g}^{-1}\right)$ was calculated using the following equation:

$$
C_{\mathrm{g}}=\frac{I \times t}{m \times \Delta V}
$$

where $I$ is the discharge current (A), $t$ is the discharge time (s), $m$ is the mass of the active material (g), and $\Delta V$ is the potential change during the discharge process $(\mathrm{V})$.

\section{Results and discussion}

Fig. 1 shows the SEM images of the as-prepared polymer particles with different P123 concentrations and $\mathrm{pH}$ values. The optimized polymer particles show a hexagonal prism-like structure with a size distribution of 200-400 nm (Fig. 1b). Thus, to gain deep understanding of morphology control, the role of $\mathrm{P} 123$ concentration and $\mathrm{pH}$ was investigated systematically (Table 1 ). The optimized mesostructured polymer (N/C-2) was obtained at a $\mathrm{pH}$ value of 9.3 with a $\mathrm{P} 123$ concentration of $32.5 \mathrm{mg} \mathrm{mL} \mathrm{m}^{-1}$, while spherical particles $(\mathrm{N} / \mathrm{C}-1, \mathrm{pH}=8.5)$ and irregular nanoparticles $(\mathrm{N} / \mathrm{C}-3, \mathrm{pH}=10.0)$ were obtained (Fig. 1a-c). This is more likely related to the polymerization rate of low molecular phenol-melamine/formaldehyde resin $(\mathrm{P}-\mathrm{M} / \mathrm{F})$, determined by the concentration of the $\mathrm{NaOH}$ catalyst. Fig. 1d and e display the morphology changes derived from the altered concentration of $\mathrm{P} 123$ with the optimized $\mathrm{pH}$ value of 9.3. As can be clearly seen, the morphology of the polymer particles was changed from spherical (N/C-4) to hexagonal prism (N/C-2) and finally to irregular cross-linked particles (N/C-5) with the increase of P123 concentration. As evidenced by small angle X-ray scattering (SAXS) analysis (Fig. S1 $\uparrow$ ), the SAXS pattern of the optimized polymer particles (N/C-2) showed an obvious peak, while a less ordered mesostructure of polymer particles was confirmed for non-optimized polymers (i.e., N/C-1, -3, -4, and -5).

SEM and TEM images in Fig. 2a-c, S2a, b, and S3a, b, $\dagger$ show the calcined N/C-2 samples at different temperatures. Regardless of the calcination temperature, the morphology and mesostructures are well maintained, indicating remarkable thermal stability of the carbon framework of OMNC. After the removal of P123 micelles during carbonization, the TEM images reveal the ordered mesopores for OMNC-700, OMNC-800, and OMNC-900 with a size of ca. 2.7, 3.6, and $3.0 \mathrm{~nm}$ (Fig. 2b, c, S2b, and S3b $\dagger$ ), respectively. From the TEM observation of individual particles (Fig. S4 $\dagger$ ), it was clearly seen that the tubular mesochannels are running in the same direction within one particle, which can act as diffusion pathways for the electrolytes. As seen in Fig. 2d-f, S2c, d, and S3c, $\mathrm{d}, \dagger$ the original morphology and mesostructures were maintained even after $\mathrm{KOH}$ activation at high temperature. The retention of 2D hexagonal mesostructures was also confirmed by SAXS patterns (Fig. 3a and S5a $\dagger$ ), further demonstrating the excellent structural stability of carbon walls between mesopores. Notably, it is ordinary to achieve the irregularly shaped ordered mesoporous carbon (OMC) with 2D hexagonal symmetry, ${ }^{31-33}$ while the hexagonal ordered mesoporous nitrogen-doped carbon (OMNC) particles have been rarely reported.

The SAXS patterns in Fig. 3a and S5a† show three resolved peaks which can be indexed to (10), (11) and (20) reflections. Compared with N/C-2 (Fig. 1b), the (10) peaks are shifted to higher values because of the polycondensation of $\mathrm{P}-\mathrm{M} / \mathrm{F}$ resin and the shrinkage of the carbon framework after calcination. As listed in Table 2, the $d_{10}$ spacing reduces from $12.17 \mathrm{~nm}(\mathrm{~N} / \mathrm{C}-2)$ to $8.85 \mathrm{~nm}$ (OMNC-700), $8.44 \mathrm{~nm}$ (OMNC-800), and $8.39 \mathrm{~nm}$ (OMNC-900) with increasing calcination temperature from 

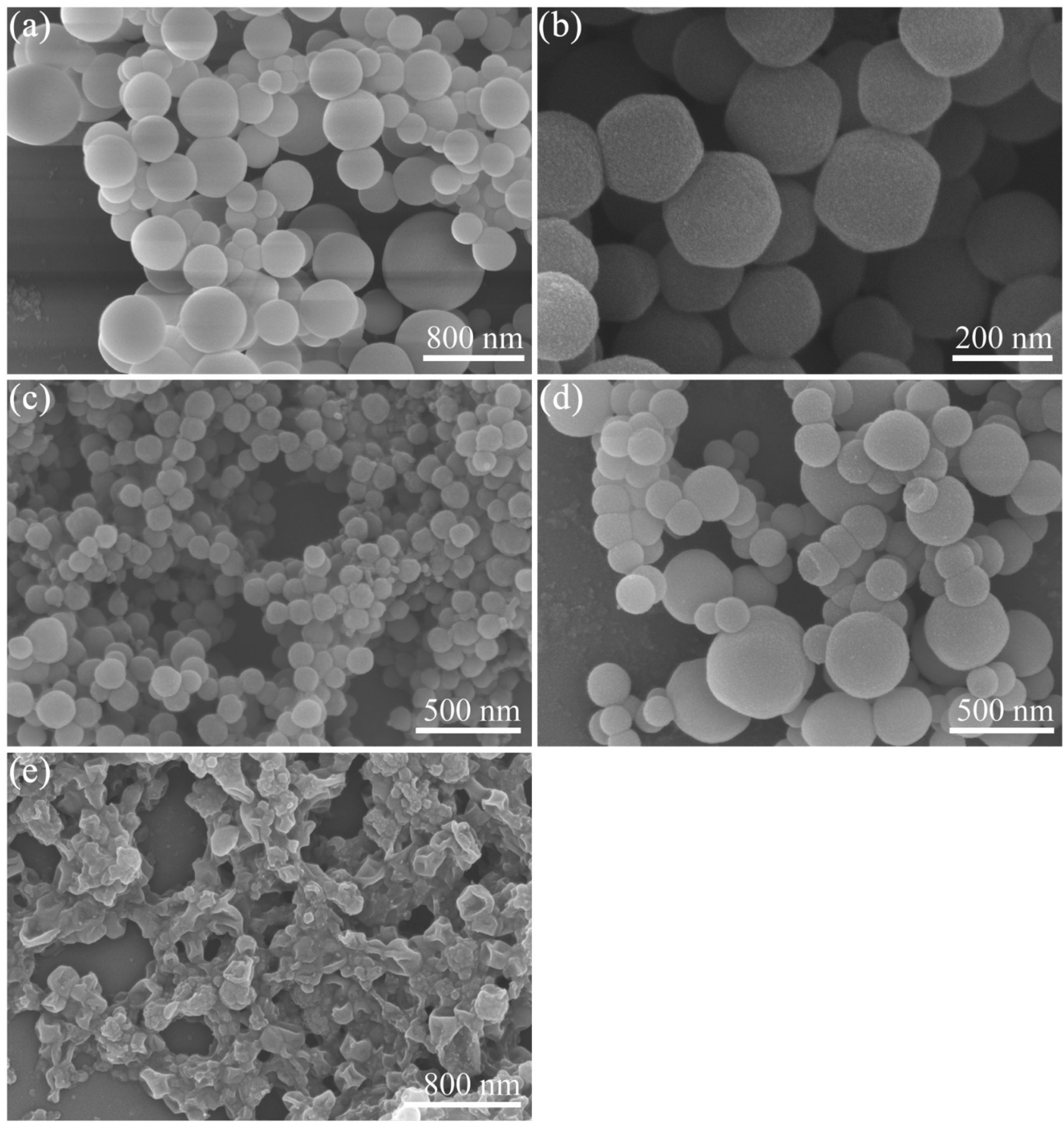

Fig. 1 (a-c) SEM images of the as prepared polymer particles with $\mathrm{P} 123$ concentration of $32.5 \mathrm{mg} \mathrm{mL}^{-1}$ at different $\mathrm{pH}$ values [(a) N/C-1 (pH $\left.=8.5\right)$, (b) $\mathrm{N} / \mathrm{C}-2(\mathrm{pH}=9.3)$ and (c) N/C-3 ( $\mathrm{pH}=10.0)$ ]. (d and e) SEM images of the as prepared polymer particles using different concentrations of $\mathrm{P} 123$ at a pH value of 9.3 [(d) N/C-4 (10.0 $\left.\mathrm{mg} \mathrm{mL}^{-1}\right)$ and (e) N/C-5 (100.0 $\left.\left.\mathrm{mg} \mathrm{mL}^{-1}\right)\right]$.

700 to $900{ }^{\circ} \mathrm{C}$. After activation with $\mathrm{KOH}$, as seen in Fig. 3a and $\mathrm{S} 5 \mathrm{a}, \uparrow$ SAXS patterns show reduced intensity of activated samples, especially for OMNC-900, indicating the deterioration of the ordered mesoporous structure due to the etching of carbon walls and the new generation of micropores. ${ }^{32,33}$

The wide-angle X-ray diffraction (XRD) patterns of all samples show two characteristic graphitic peaks located at around $23^{\circ}$ and $44^{\circ}$ which can be assigned to (002) and (101) planes (Fig. 3b and S5 $\mathrm{b}_{\dagger} \dagger$ ), respectively. After $\mathrm{KOH}$ activation, the peak of the (002) plane disappeared except for OMNC-900A because OMNC-900 pre-carbonized at a higher temperature was more stable than OMNC-700 and OMNC-800 during activation. This variation should be ascribed to the etching reaction between the carbon framework and $\mathrm{KOH}$ 

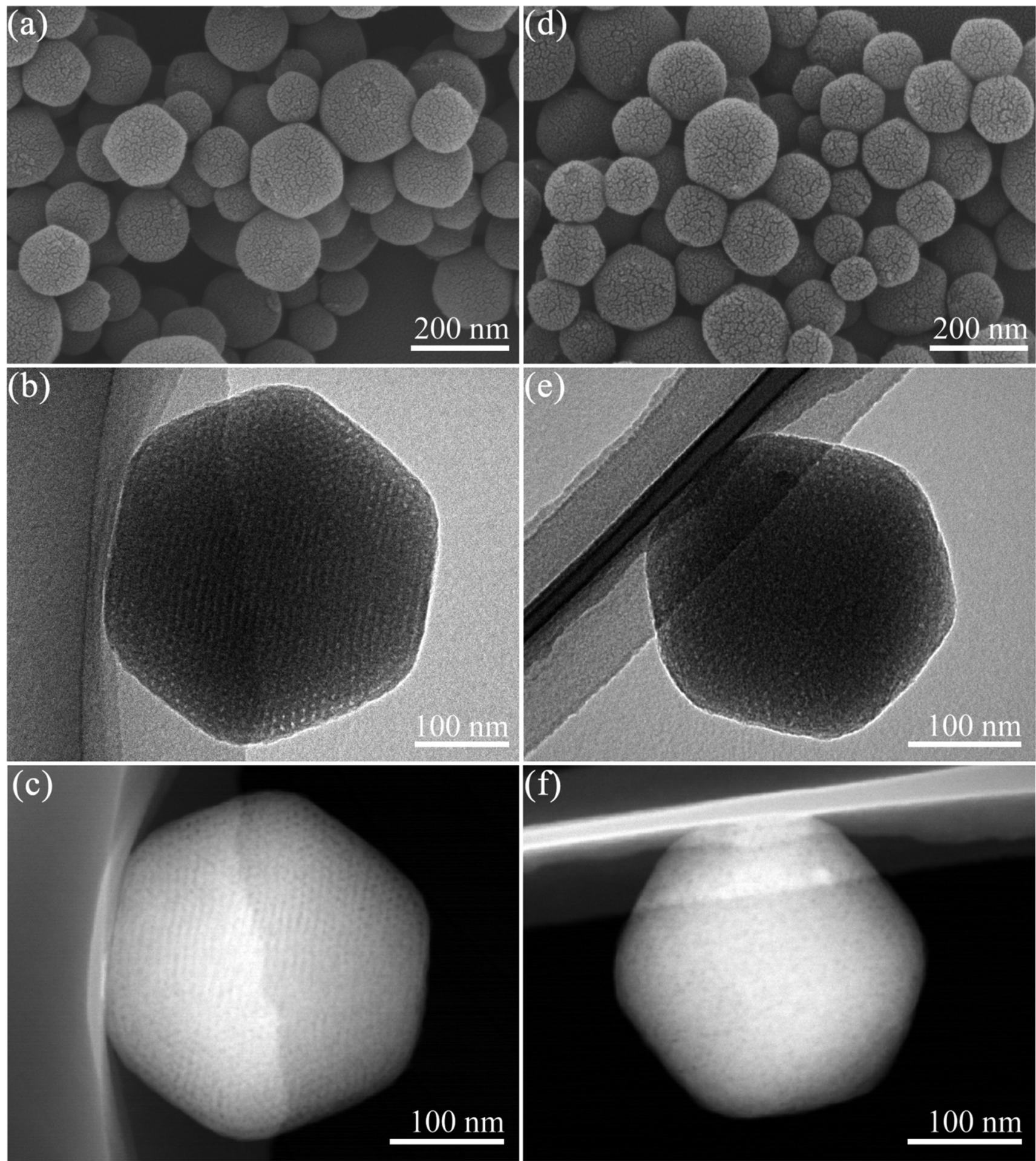

Fig. 2 SEM, TEM, and HAADF-STEM images of representative samples: (a-c) OMNC-800 carbonized at $800{ }^{\circ} \mathrm{C}$; (d-f) corresponding activated product OMNC-800A.

which consumed the carbon skeleton and created more pores. ${ }^{34,35}$

High resolution X-ray photoelectron spectroscopy (XPS) was conducted for the representative OMNC-800 and activated OMNC-800A (Fig. 3c) to characterize the chemical state of nitrogen. The N 1s XPS spectra of OMNC-800 and OMNC-800A were deconvoluted into four peaks at $398.2 \mathrm{eV}$ (pyridinic N), $399.5 \mathrm{eV}$ (pyrrolic N), $400.9 \mathrm{eV}$ (graphitic N), and $403.2 \mathrm{eV}$ (oxidized $\mathrm{N}$ ). ${ }^{36,37}$ The $\mathrm{N}$ content of 6.5 at\% (OMNC-800) was decreased to 0.9 at $\%$ (OMNC-800A) after $\mathrm{KOH}$ activation due to 

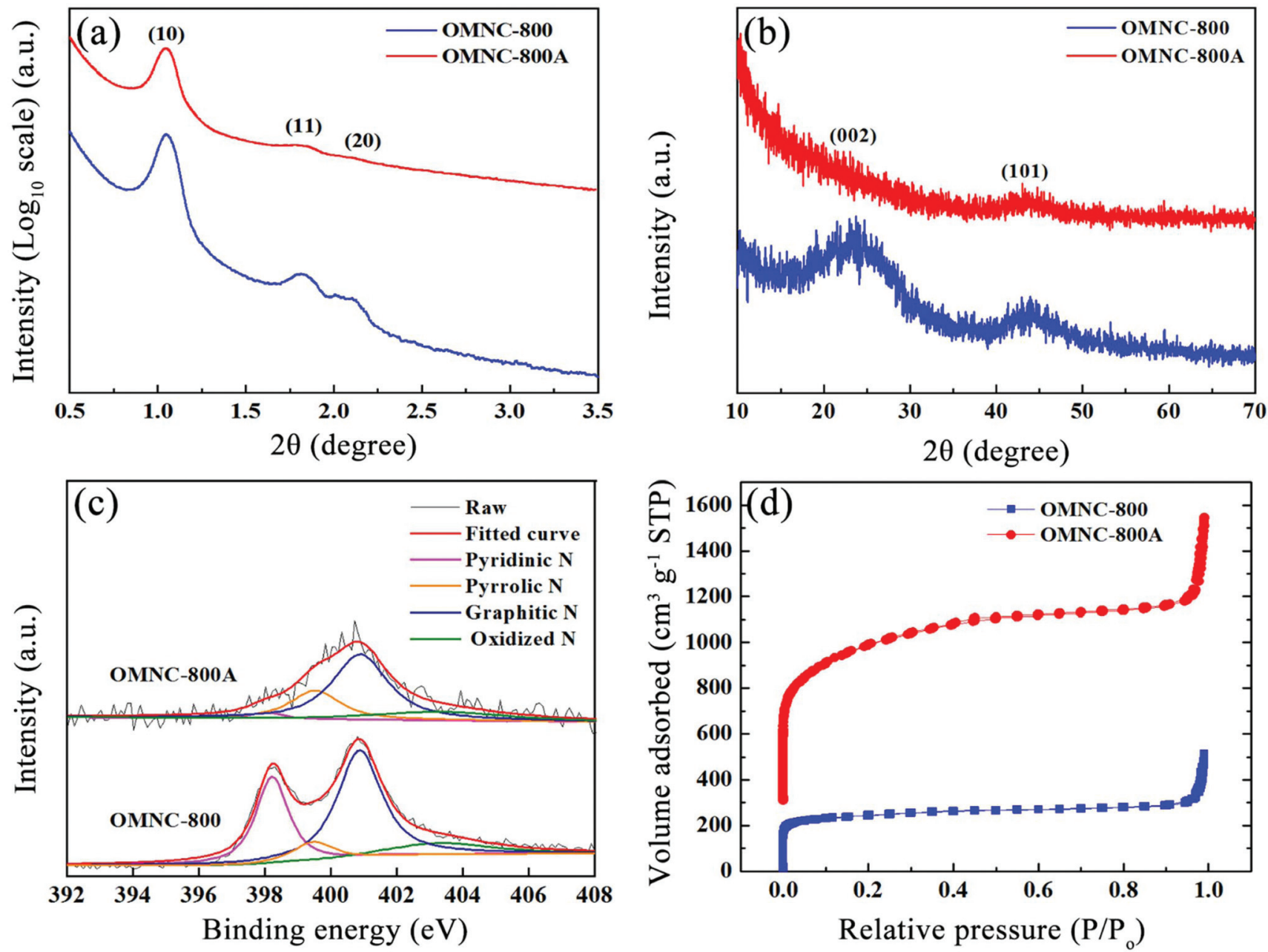

Fig. 3 (a) SAXS patterns, (b) XRD patterns, (c) high-resolution N 1s XPS spectra, and (d) $\mathrm{N}_{2}$ adsorption-desorption isotherms of OMNC-800 and OMNC-800A.

Table 2 Physicochemical properties of OMNC- $T$ and OMNC-TA $(T=$ $700,800,900)$

\begin{tabular}{lccl}
\hline Sample & $d_{10}(\mathrm{~nm})$ & $\begin{array}{l}\text { Specific surface } \\
\text { area }\left(\mathrm{m}^{2} \mathrm{~g}^{-1}\right)\end{array}$ & $\begin{array}{l}\text { Pore volume } \\
\left(\mathrm{cm}^{3} \mathrm{~g}^{-1}\right)\end{array}$ \\
\hline N/C-2 & 12.17 & - & - \\
OMNC-700 & 8.85 & 696 & 0.37 \\
OMNC-800 & 8.44 & 840 & 0.49 \\
OMNC-900 & 8.39 & 893 & 0.45 \\
OMNC-700A & 8.57 & 1790 & 0.89 \\
OMNC-800A & 8.41 & 2450 & 1.34 \\
OMNC-900A & 8.14 & 1630 & 0.93
\end{tabular}

partial replacement of nitrogen by oxygen through the activation reaction. ${ }^{38}$ The relative content of all $\mathrm{N}$ species in OMNC-800 and OMNC-800A was estimated by XPS analysis and is summarized in Fig. S6. $\dagger$ After $\mathrm{KOH}$ activation, the percentage of pyridinic $\mathrm{N}$ dropped from $29.43 \%$ for OMNC-800 to $4.65 \%$ for OMNC-800A. In contrast, the percentage of graphitic-N increased from $49.60 \%$ for OMNC-800 to $53.96 \%$ for OMNC-800A. These findings are consistent with the fact that graphitic $\mathrm{N}$ is more stable than pyridinic $\mathrm{N} .{ }^{39}$ In general, pyridinic $\mathrm{N}$ and graphitic $\mathrm{N}$ in carbons are regarded as electroactive sites, which would benefit the enhancement of electrical conductivity as well as capacitances. ${ }^{40,41}$

Based on the analysis of $\mathrm{N}_{2}$ adsorption-desorption isotherms (Fig. 3d and S5c $\dagger$ ), the increase of the calcination temperature from 700 to 800 and $900{ }^{\circ} \mathrm{C}$ led to the gradual increase in the specific surface area (SSA) from 696 to 840 and $893 \mathrm{~m}^{2} \mathrm{~g}^{-1}$, respectively. The $\mathrm{KOH}$ activation process caused a significant enhancement of the SSA for all activated samples, as shown in Table 2. The much higher $\mathrm{N}_{2}$ uptake at a low relative pressure $\left(P / P_{0}<0.1\right)$ for activated products (OMNC-TA) can be clearly observed, which indicates the formation of tremendous micropores after activation. The SSA of OMNC-800A is obviously higher than that of other activated samples, reaching up to $2450 \mathrm{~m}^{2} \mathrm{~g}^{-1}$. This was attributed to the highest pore volume of OMNC-800 which was beneficial to the deep impregnation of molten $\mathrm{KOH}$. In addition, as mentioned above, OMNC-900 calcined at high temperature was more difficult to activate deeply by $\mathrm{KOH}$. 
Considering the unique structural advantages of OMNC- $T$, including well-dispersed particles, uniformly sized mesopores, tubular mesochannels, and high surface area, it is expected to be a promising electrode material for practical supercapacitors. To show the superiority of OMNC-800A, the electrochemical performances of OMNC- $T$ and OMNC-800A were investigated in a three-electrode system using $1 \mathrm{M} \mathrm{H}_{2} \mathrm{SO}_{4}$ as the electrolyte. CV curves shown in Fig. 4a indicate that all samples display rectangle-like curves, revealing the typical electrical double layer capacitive properties of all samples. Remarkably, OMNC-800 exhibited a higher CV area than OMNC-700 and OMNC-900, suggesting that OMNC-800 has a better capacitive property compared to OMNC-700 and OMNC-900. In addition, after activation with $\mathrm{KOH}$, the obtained OMNC-800A showed an improved capacitive performance compared to pristine OMNC-800. The specific capacitances at $0.5 \mathrm{~A} \mathrm{~g}^{-1}$ (denoted as $C_{0.5}$ ) were calcu- lated by GCD curves (Fig. 4b) for all samples (OMNC- $T$ and OMNC-800A). The corresponding values of $C_{0.5}$ are 196.81 (OMNC-700), 332.12 (OMNC-800), 314.56 (OMNC-900), and $475.75 \mathrm{~F} \mathrm{~g}^{-1}$ (OMNC-800A). Obviously, OMNC-800A displayed the highest specific capacitance of $475.75 \mathrm{~F} \mathrm{~g}^{-1}$. To the best of our knowledge, this value is also higher than those for all the reported $\mathrm{N}$-doped carbon values measured in an aqueous electrolyte utilizing a three-electrode system (Table S1†). In addition, the GCD plots shown in Fig. 4b are generally symmetric, which is indicative of high capacitive reversibility and very low internal resistance of all samples.

Fig. 4c shows the specific capacitances of OMNC- $T$ and OMNC-800A calculated based on the discharge curves at various current densities from 0.5 to $10 \mathrm{~A} \mathrm{~g}^{-1}$. As expected, the capacitance values of OMNC-800A surpass those of nonactivated samples at any current density. In addition, even at a relatively high current density of $10 \mathrm{~A} \mathrm{~g}^{-1}$, the capacitance
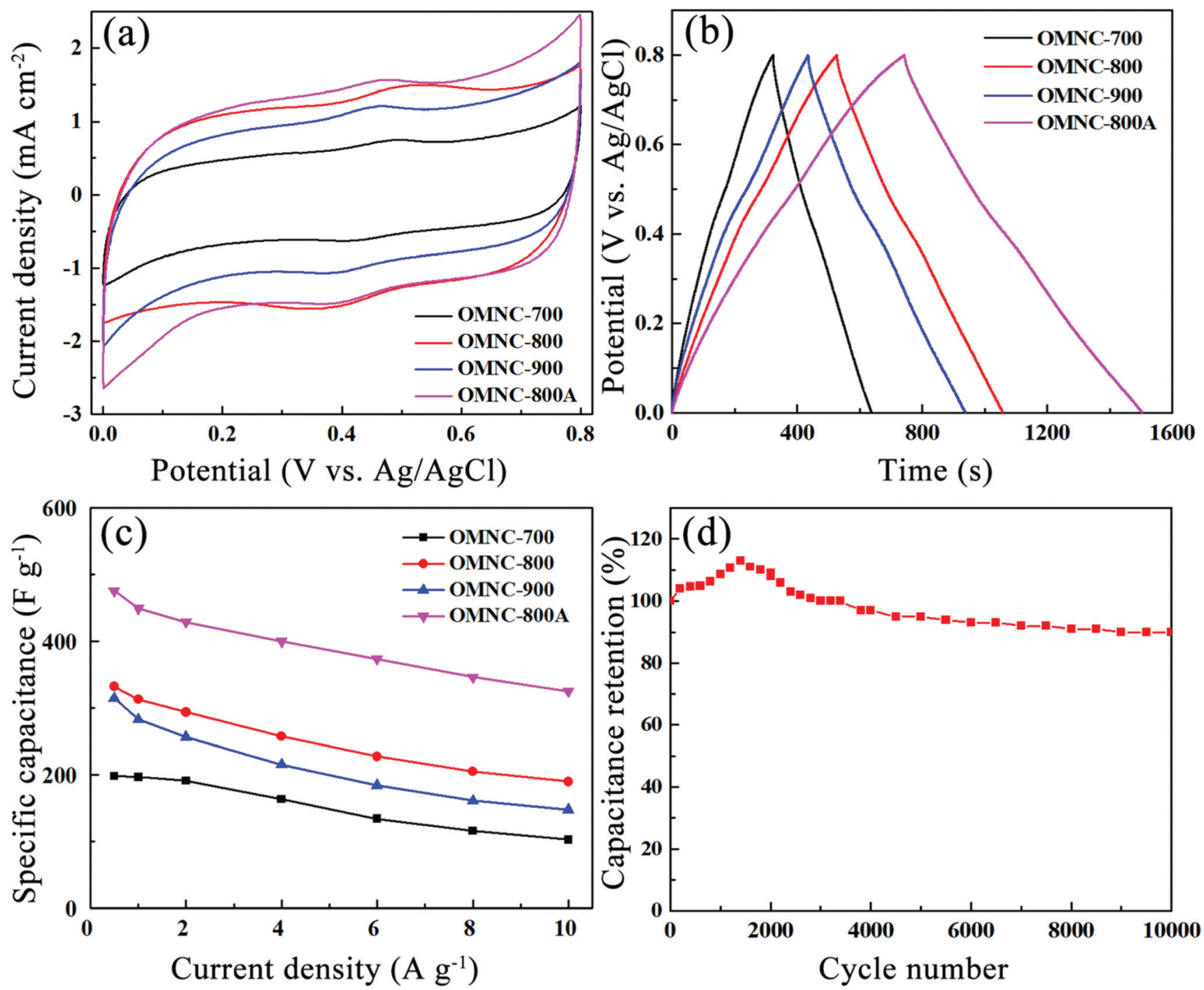

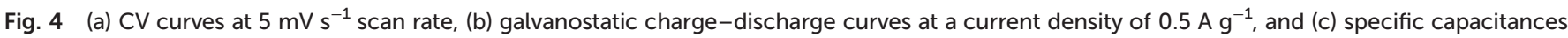
at different current densities for OMNC-T $(T=700,800,900)$ and OMNC-800A; (d) cycling performance of OMNC-800A at $100 \mathrm{mV} \mathrm{s}^{-1}$. 
value of OMNC-800A as high as $325 \mathrm{~F} \mathrm{~g}^{-1}$ (Fig. S7 $\dagger$ ), displaying a remarkable rate capacitance retention (68\%). Such excellent capacitance retention is an indication for quick charge propagation capability and easy ion transport within the OMNC-800A electrode material. To further demonstrate the superiority of OMNC-800A, the long-term stability was investigated by the $\mathrm{CV}$ measurement at $100 \mathrm{mV} \mathrm{s}^{-1}$ (Fig. 4d and S8†). Remarkably, the capacitance for the first 2000 cycles gradually increased until capacitance retention reached $113 \%$, possibly due to the activation process of the OMNC-800A electrode by improved wetting of the electrolyte deep inside the mesochannels of the material. After 10000 cycles, it retained $90 \%$ of its initial capacitance value, indicating a good electrode stability.

The above results indicate that OMNC-800A is a promising carbon material for supercapacitors. The superior capacitive performance can be ascribed to the following reasons: (i) OMNC-800A exhibited an ultrahigh specific surface area and a large pore volume $\left(2450 \mathrm{~m}^{2} \mathrm{~g}^{-1}\right.$ and $\left.1.34 \mathrm{~cm}^{3} \mathrm{~g}^{-1}\right)$. As is well known, the high specific surface area and large pore volume of porous carbons usually result in high electrochemical double layer capacitance. ${ }^{42,43}$ (ii) OMNC-800A exhibited a novel hierarchically porous structure with plentiful mesochannels, not only guaranteeing the electrode wetting by the electrolyte, but also help to accelerate the ion transport.

\section{Conclusion}

In this study, we demonstrated the preparation of mesostructured polymer particles by a soft-templating approach using a P123 triblock copolymer. Phenol-melamine/formaldehyde resin was used as the carbon precursor and nitrogen source. After carbonization, OMNC materials with 2D hexagonal mesostructures were obtained. The further $\mathrm{KOH}$-activated OMNC maintained the original 2D hexagonal mesostructures. Activated OMNC-800A featured a high specific surface area $\left(2450 \mathrm{~m}^{2} \mathrm{~g}^{-1}\right)$ and revealed an outstanding gravimetric specific capacitance of 475.75 $\mathrm{F} \mathrm{g}^{-1}$ at a current density of $0.5 \mathrm{~A} \mathrm{~g}^{-1}$ and excellent stability with high capacitance retention in a three-electrode system. Moreover, the salient features of the synthetic approach developed in this study are simplicity, environmental friendliness, and reliability, which are beneficial to mass production for practical application in supercapacitors.

\section{Conflicts of interest}

There are no conflicts to declare.

\section{Acknowledgements}

This work was supported by an Australian Research Council (ARC) Future Fellow (grant no. FT150100479), JSPS KAKENHI (grant no. 17H05393 and 17K19044), and the research fund by the Suzuken Memorial Foundation. This work was also sup- ported by the Deanship of Scientific Research (DSR), King Abdulaziz University, Jeddah (KEP-7-130-39). Abeer Enaiet Allah appreciates the financial support by joint supervision scholarship from Cultural affairs and missions sector, Egyptian Ministry of Higher Education (MOHE).

\section{References}

1 C. Liang, Z. Li and S. Dai, Angew. Chem., Int. Ed., 2008, 47, 3696-3717.

2 T. Y. Ma, L. Liu and Z.-Y. Yuan, Chem. Soc. Rev., 2013, 42, 3977-4003.

3 F. Su, J. Zeng, X. Bao, Y. Yu, J. Y. Lee and X. S. Zhao, Chem. Mater., 2005, 17, 3960-3967.

4 B. Guo, X. Wang, P. F. Fulvio, M. Chi, S. M. Mahurin, X.-G. Sun and S. Dai, Adv. Mater., 2011, 23, 4661-4666.

5 G. Shen, X. Sun, H. Zhang, Y. Liu, J. Zhang, A. Meka, L. Zhou and C. Yu, J. Mater. Chem. A, 2015, 3, 24041-24048.

6 S. Jun, S. H. Joo, R. Ryoo, M. Kruk, M. Jaroniec, Z. Liu, T. Ohsuna and O. Terasaki, J. Am. Chem. Soc., 2000, 122, 10712-10713.

7 S. Leyva-García, D. Lozano-Castelló, E. Morallón and D. Cazorla-Amorós, J. Mater. Chem. A, 2016, 4, 4570-4579.

8 J. Lee, S. Yoon, T. Hyeon, S. M. Oh and K. B. Kim, Chem. Commun., 1999, 2177-2178.

9 D. Liu, L. J. Xia, D. Qu, J. H. Lei, Y. Li and B. L. Su, J. Mater. Chem. A, 2013, 1, 15447-15458.

10 W. C. Chu, B. P. Bastakoti, Y. V. Kaneti, J. G. Li, H. R. Alamri, Z. A. Alothman, Y. Yamauchi and S. W. Kuo, Chem. - Eur. J., 2017, 23, 13734-13741.

11 J. Tang, J. Liu, C. Li, Y. Li, M. O. Tade, S. Dai and Y. Yamauchi, Angew. Chem., Int. Ed., 2015, 54, 588593.

12 J. Tang, J. Liu, R. R. Salunkhe, T. Wang and Y. Yamauchi, Chem. Commun., 2016, 52, 505-508.

13 H. Tan, Y. Li, X. Jiang, J. Tang, Z. Wang, H. Qian, P. Mei, V. Malgras, Y. Bando and Y. Yamauchi, Nano Energy, 2017, 36, 286-294.

14 A. Eftekhari and Z. Fan, Mater. Chem. Front., 2017, 1, 10011027.

15 E. Lim, C. Jo and J. Lee, Nanoscale, 2016, 8, 78277833.

16 X. Xu, J. Tang, H. Qian, S. Hou, Y. Bando, M. S. A. Hossain, L. Pan and Y. Yamauchi, ACS Appl. Mater. Interfaces, 2017, 9, 38737-38744.

17 R. R. Salunkhe, Y. H. Lee, K. H. Chang, J. M. Li, P. Simon, J. Tang, N. L. Torad, C. C. Hu and Y. Yamauchi, Chem. Eur. J., 2014, 20, 13838-13852.

18 X. Yu, J. Wang, Z.-H. Huang, W. Shen and F. Kang, Electrochem. Commun., 2013, 36, 66-70.

19 M. Li, J. Ding and J. Xue, J. Mater. Chem. A, 2013, 1, 74697476.

20 J. Tang, J. Wang, L. K. Shrestha, M. S. A. Hossain, Z. A. Alothman, Y. Yamauchi and K. Ariga, ACS Appl. Mater. Interfaces, 2017, 9, 18986-18993. 
21 J. Huang, B. G. Sumpter and V. Meunier, Chem. - Eur. J., 2008, 14, 6614-6626.

22 L. Borchardt, M. Oschatz and S. Kaskel, Mater. Horiz., 2014, 1, 157-168.

23 L. Zhang, X. Yang, F. Zhang, G. Long, T. Zhang, K. Leng, Y. Zhang, Y. Huang, Y. Ma, M. Zhang and Y. Chen, J. Am. Chem. Soc., 2013, 135, 5921-5929.

24 T. Lin, I.-W. Chen, F. Liu, C. Yang, H. Bi, F. Xu and F. Huang, Science, 2015, 350, 1508-1513.

25 A. J. R. Rennie, V. L. Martins, R. M. Smith and P. J. Hall, Sci. Rep., 2016, 6, 22062.

26 F. Zhang, D. Gu, T. Yu, F. Zhang, S. Xie, L. Zhang, Y. Deng, Y. Wan, B. Tu and D. Zhao, J. Am. Chem. Soc., 2007, 129, 7746-7747.

27 D. Gu, H. Bongard, Y. Meng, K. Miyasaka, O. Terasaki, F. Zhang, Y. Deng, Z. Wu, D. Feng, Y. Fang, B. Tu, F. Schüth and D. Zhao, Chem. Mater., 2010, 22, 4828-4833.

28 J. Yu, M. Guo, F. Muhammad, A. Wang, G. Yu, H. Ma and G. Zhu, Microporous Mesoporous Mater., 2014, 190, 117-127.

29 D. Liu, C. Zeng, D. Qu, H. Tang, Y. Li, B. L. Su and D. Qu, J. Power Sources, 2016, 321, 143-154.

30 A. Chen, Y. Yu, Y. Zhang, W. Zang, Y. Yu, Y. Zhang, S. Shen and J. Zhang, Carbon, 2014, 80, 19-27.

31 J. Zhu, J. Yang, R. Miao, Z. Yao, X. Zhuang and X. Feng, J. Mater. Chem. A, 2016, 4, 2286-2292.

32 Y. Lv, F. Zhang, Y. Dou, Y. Zhai, J. Wang, H. Liu, Y. Xia, B. Tu and D. Zhao, J. Mater. Chem., 2012, 22, 93-99.
33 W. Teng, Z. Wu, J. Fan, H. Chen, D. Feng, Y. Lv, J. Wang, A. M. Asiri and D. Zhao, Energy Environ. Sci., 2013, 6, 27652776.

34 R. Heimböckel, S. Kraas, F. Hoffmann and M. Fröba, Appl. Surf. Sci., 2018, 427, 1055-1064.

35 F. Sun, J. Gao, X. Liu, X. Pi, Y. Yang and S. Wu, Appl. Surf. Sci., 2016, 387, 857-863.

36 I. Kusunoki, M. Sakai, Y. Igari, S. Ishidzuka, T. Takami, T. Takaoka, M. Nishitani-Gamo and T. Ando, Surf. Sci., 2001, 492, 315-328.

37 E. Raymundo-Piñero, D. Cazorla-Amorós, A. LinaresSolano, J. Find, U. Wild and R. Schlögl, Carbon, 2002, 40, 597-608.

38 M. Wahid, G. Parte, D. Phase and S. Ogale, J. Mater. Chem. A, 2015, 3, 1208-1215.

39 Y. Chen, Q. Liu and J. Wang, Nano Adv., 2016, 1, 7989.

40 R. Czerw, M. Terrones, J. C. Charlier, X. Blase, B. Foley, R. Kamalakaran, N. Grobert, H. Terrones, D. Tekleab, P. M. Ajayan, W. Blau, M. Rühle and D. L. Carroll, Nano Lett., 2001, 1, 457-460.

41 Y. Deng, Y. Xie, K. Zou and X. Ji, J. Mater. Chem. A, 2016, 4, 1144-1173.

42 J. Chmiola, G. Yushin, Y. Gogotsi, C. Portet, P. Simon and P. L. Taberna, Science, 2006, 313, 1760-1763.

43 L. L. Zhang and X. S. Zhao, Chem. Soc. Rev., 2009, 38, 25202531. 